Reprod. Nutr. Dévelop., 1988, 28 (6 B), 1655-1672.

\title{
Protéines trophoblastiques
}

J. MARTAL, Nicole CHÊNE, Madia CHARLIER, G. CHARPIGNY, Sylvaine CAMOUS, M. GUILLOMOT, Pierrette REINAUD, J. BERTIN, P. HUMBLOT ( ${ }^{*}$ )

I.N.R.A., Unité d'Endocrinologie de l'Embryon.

Station de Physiologie animale, 78350 Jouy-en-Josas, France

(") U.N.C.E.I.A., Laboratoire d'Hormonologie, B.P. 65 ,

94703 Maisons-Alfort, France

\section{Summary. Trophoblastic proteins.}

Many studies conducted on human or animal placenta (chorion) suggest that the trophoblast is not only a passive filter between maternal and foetal blood flow, but is also endowed with complex functions. Factors of trophoblast origin involved in the mechanism of pregnancy recognition or maintenance of the progesterone environment required for the embryo survival, are reviewed. The main proteins involved in pregnancy are reported in table 1. Emphasis is laid on early signals of pregnancy which are of practical interest in human clinical medicine and animal husbandry. Among them, human chorionic gonadotropin (hCG), protein SP1 ("Schwangerschaftsprotein» 1) and some pregnancyassociated plasma proteins such as the PAPP A are very useful in the diagnosis of pregnancy, abortion, foetal abnormality or tumor in the human species. The presence of trophoblastin (presently studied in our laboratory) of a pregnancy-specific protein $B$ and of early pregnancy factor (EPF) attest the establishment of pregnancy in domestic animals and in other mammals. The biological properties of some hormones such as placental lactogens (PL) or chorionic somatomammotropins (CS), human placental growth hormone $(\mathrm{hPGH})$ contribute to a better understanding of the gestation function. Many other factors participate in the foetal development, for example, proliferin. Some proteins can display immunosuppressive properties or be responsible for the immune tolerance between the mother and the foetus. Although many placental proteins have already been defined, their biological functions have not yet been elucidated.

De nombreux travaux conduits à partir de placenta (chorion) humain ou animal ont suggéré que le rôle du trophoblaste ne se limitait pas seulement à celui d'un filtre passif entre la circulation maternelle et fotale, mais qu'il remplissait des fonctions complexes. Outre les synthèses protéiques comprenant les sécrétions d'enzymes, de proenzymes, d'activateurs et d'inhibiteurs enzymatiques et de protéines structurales qui sont produites au sein du trophoblaste, celui-ci assure également la sécrétion d'hormones stéroïdes et protéiques. Le trophoblaste se comporte donc comme un organe à part entière, capable d'assurer la croissance du fœtus, mais aussi de synthétiser et de sécréter différentes hormones à la manière d'une glande endocrine. Par trophoblaste, nous sous-entendrons, dans l'espèce humaine (à placentation hémochoriale) le cytotrophoblaste et surtout le syncytiotrophoblaste et dans les espèces à placentation épithéliochoriale, les 
feuillets constitués par l'endoderme et le trophectoderme et le chorion (cotylédons fœtaux chez les ruminants). Parmi les substances biologiquement actives et spécifiques, les protéines placentaires occupent une place importante en raison de leurs multiples activités. Certaines d'entre elles rendent compte de l'établissement de la gestation et à cet égard, elles sont très utiles dans la détermination du diagnostic précoce de gestation. C'est le cas de l'hCG (gonadotrophine chorionique humaine), qui fut la première hormone placentaire détectée en 1927 par Aschheim et Zondek. Une protéine très prometteuse la PSPB (pregnancy specific protein $B$ ) pour un diagnostic précoce de gestation chez le bovin a été mise en évidence (Butler et al., 1982 ; Sasser et al., 1985). D'autres protéines participent au contrôle de l'environnement progestéronique de l'utérus. Parmi celles-ci, citons de nouveau l'hCG qui stimule la sécrétion de progestérone et permet le maintien du " corpus luteum graviditatis ». Chez la brebis, une protéine, la trophoblastine, intervient dans le maintien du corps jaune de gestation (Martal et al., 1979; Godkin et al., 1984b). Le développement embryonnaire peut être régulé par différentes hormones placentaires, telles que les hormones lactogènes placentaires $(\mathrm{PL})$ ou somatomammotropines chorioniques (CS) et par une hormone de croissance placentaire, récemment décrite par Hennen et al. (1985) dans l'espèce humaine ( $\mathrm{hPGH}$ ). De nombreux autres facteurs interviennent dans le développement ; citons par exemple la proliférine, identifiée chez la souris par Linzer et Nathans (1984). Certaines protéines peuvent présenter des propriétés immunosuppressives ou sont responsables de la tolérance immunitaire du couple mère-fœtus. Bien que nombre de protéines placentaires soient bien définies, il reste souvent encore à déterminer leurs fonctions biologiques.

L'ensemble des principales protéines impliquées dans la gestation est consigné dans le tableau 1.

Dans cet article, nous nous limiterons à l'étude de quelques protéines en fonction de leur originalité ou de l'intérêt qu'elles suscitent, par exemple dans le diagnostic de gestation précoce ou de la viabilité embryonnaire.

\section{A. Protéines impliquées dans l'établissement de la gestation ou signaux embryonnaires de reconnaissance de la gestation.}

CG. - La gonadotrophine chorionique humaine (hCG) apparaît dans l'urine de femme enceinte dès le $5^{\mathrm{e}}$ jour après la fécondation. Son rôle physiologique reste encore à éclaircir (voir revue de Gaspard, 1979). Cependant, I'hCG synthétisée par le syncytiotrophoblaste (Midgley et Pierce, 1962) stimule la sécrétion de progestérone par le corps jaune gestatif et permet ainsi l'établissement de la grossesse (Yoshimi et al., 1969). L'intérêt principal de la détection de I'hCG réside dans le diagnostic de gestation précoce, le dépistage des menaces d'avortement, ainsi que celui de certaines tumeurs. La caractérisation de l'hCG est réalisée au moyen de tests biologiques et surtout immunologiques (G-test).

Une gonadotrophine chorionique est également présente, en abondance, au cours de la gestation, chez le singe (Hodgen et al.), en moindre quantité chez la souris (Wiley, 1974), la lapine (Haour et Saxena, 1974), la ratte (Haour et al., 
1976) et en très faible concentration chez la brebis (Wintenberger-Torrès, 1978 ; Lacroix et Martal, 1979). Une CG existe également chez la vache (Ailenberg etShemesh, 1983; Izhar et Shemesh, 1988). Le sérum de jument gravide recèle une importante quantité de gonadotrophine chorionique (oCG) dénommée par Cole et Hart (1930) «Pregnant Mare Serum Gonadotropin» (PMSG). Cette hormone est sécrétée par les coupes endométriales. Elle n'est présente qu'à partir du $35^{\mathrm{e}}$ jour de gestation. Elle ne peut donc, comme l'hCG, prévenir la lutéolyse en début de gestation, qui commence dès les $15-16^{\mathrm{e}}$ jours. Son rôle reste donc mal établi.

$S P 1$. - Le diagnostic de gestation précoce chez la femme peut s'apprécier par la présence d'une protéine sérique ou "Schwangerschaft protein 1 » (SP1) (Bohn, 1971). Elle peut être aussi détectée dans la phase lutéale du cycle au cours de laquelle la femme devient enceinte (Ahmed et Klopper, 1983 ; Ho et al., 1988). Le dosage radio ou enzymo-immunologique de la SP1 peut également être mis à profit dans le diagnostic de menaces d'avortement (Klopper et Ahmed, 1985) et dans la révélation de tumeurs trophoblastiques (Seppälä et al., 1978).

PSP. - La PSPB, "Pregnancy Specific Protein B », est un message sérique de reconnaissance de la gestation, partiellement caractérisé chez les bovins par Butler et al. (1982). Sa détection dans le sérum bovin à l'aide d'un anticorps, à partir du $24^{\mathrm{e}}$ jour après la conception, constitue un diagnostic spécifique de l'état gestatif (Sasser et al., 1986). En effet, le dosage de la progestérone, reflet de l'activité lutéale, constitue un excellent diagnostic de non-gestation (précision d'environ $100 \%$ ) lorsqu'il est utilisé dans des conditions appropriées ; mais il peut engendrer un certain nombre de faux diagnostics positifs de gestation, notamment dans le cas de corps jaunes persistants à la suite de mortalité embryonnaire tardive (à partir du $16^{\mathrm{e}}$ jour chez la vache). De plus, en raison de la sécrétion de progestérone durant chaque phase lutéale du cycle œstrien, il est indispensable de connaître rigoureusement la date de la saillie ou de l'insémination artificielle. L'exactitude de ce test dans le diagnostic de gestation n'est que d'environ $70 \%$.

Plus récemment, la purification d'une protéine spécifique de la gestation ( $P S P$ ou $P S_{60}$ : protéine sérique de gestation) a été réalisée à partir de cotylédons bovins prélevés au $168^{\mathrm{e}}$ jour de gestation (Camous et al., 1988). L'utilisation d'une résine échangeuse de cations, dans un système de chromatographie à haute performance (HPLC), a conduit à l'obtention d'une protéine vraisemblablement glycosylée dont le poids moléculaire apparent est d'environ 60 kdal par détermination en gel d'électrophorèse-SDS. Le point isoélectrique est estimé à 5,1-5,5. Les caractéristiques de cette protéine diffèrent donc de celles de la PSPB, qui pourrait être un mélange de molécules différentes dont un poids moléculaire apparent a été défini, compris entre 47 et $53 \mathrm{kdal}$ (Butler et al., 1982), puis à $78 \mathrm{kdal}$ (Sasser et al., 1988) et un pl de l'ordre de 4-4,4. La sécrétion de la PSPB semble assurée par les cellules binucléées du trophoblaste (Reimers et al., 1985). Elle existe également chez la brebis, la chèvre, le cerf, le mouflon et le wapiti (Sasser et al., 1985). Une étude expérimentale, réalisée par Humblot en collaboration avec Sasser et Camous (Humblot et al., 1988a), a conduit aux remarques suivantes. La mesure de la PSPB, évaluée par un test radioimmuno 


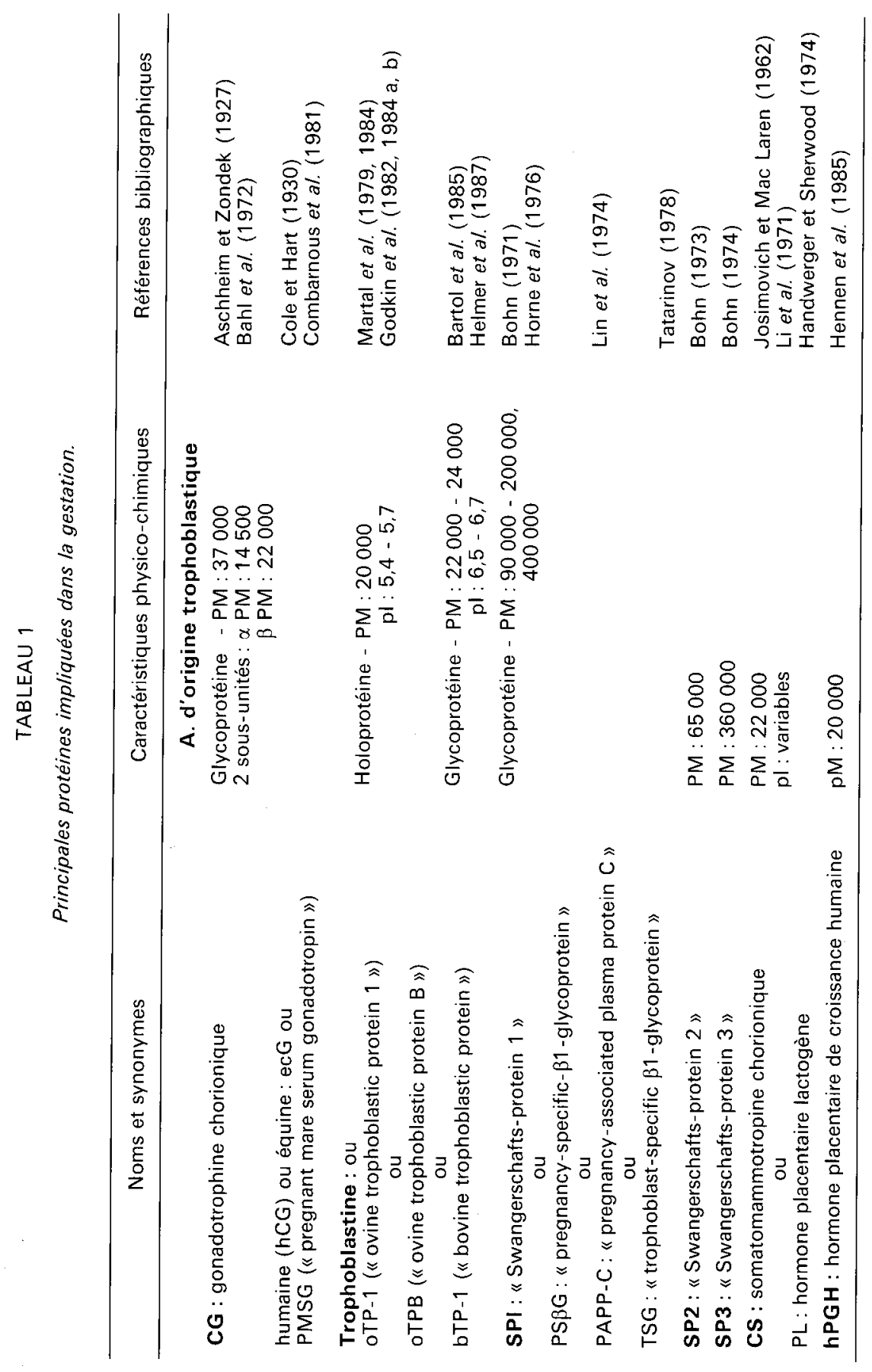




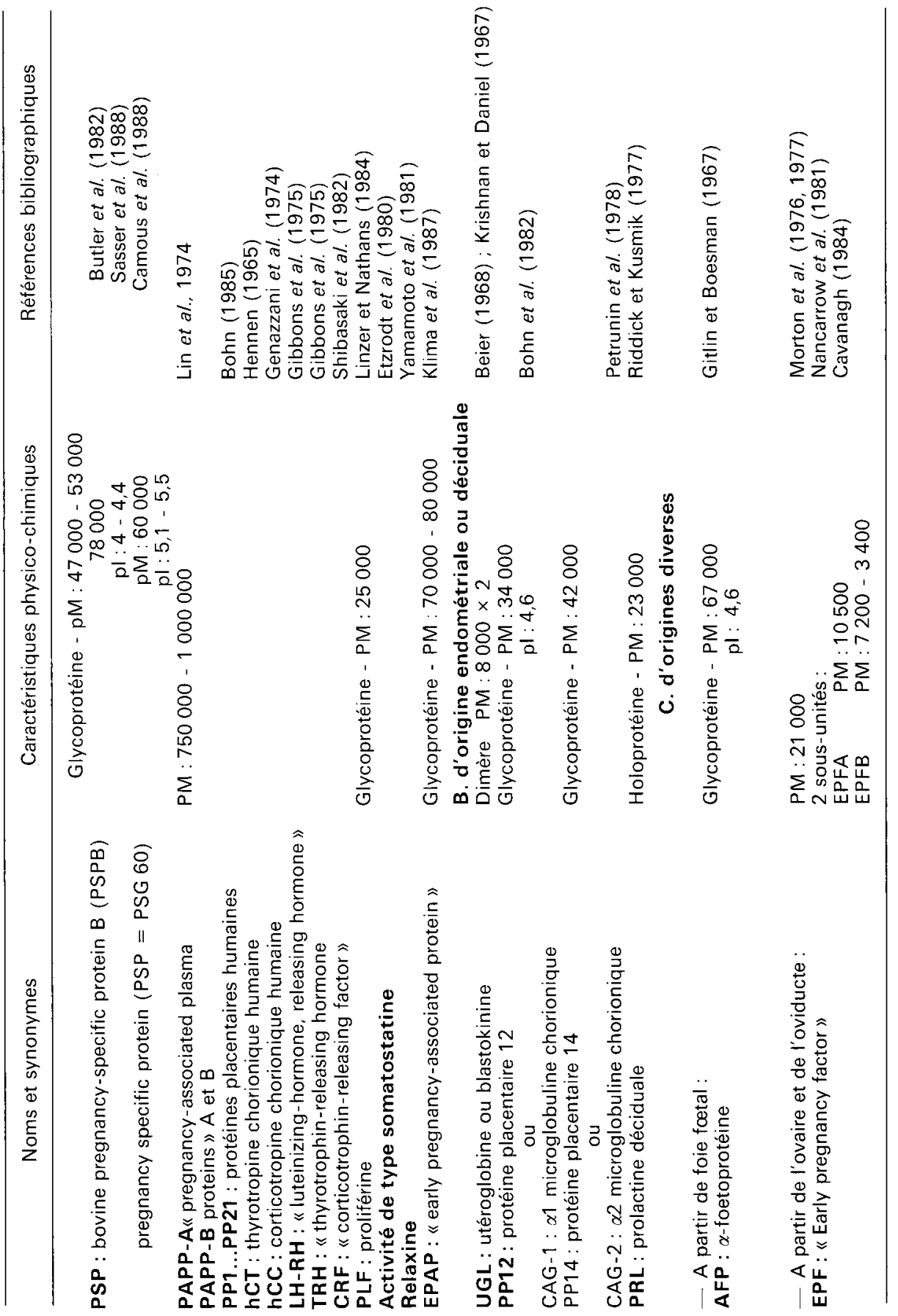


logique, constitue un excellent moyen pour suivre la mortalité embryonnaire après le $16^{e}$ jour de gestation. Le fait que les PSP soient sécrétées tout au long de la gestation et augmentent jusqu'à la fin de celle-ci rend la mesure de sa concentration très aisée, surtout à partir du $30^{\circ}$ jour et confère au diagnostic de gestation précoce une précision acceptable. Toutefois, pour que cette méthode de diagnostic soit suffisamment fiable (à $99 \%$ ), il est nécessaire d'observer un délai de 70 jours entre la précédente mise-bas de la vache et sa nouvelle insémination, afin d'éviter les interférences des taux résiduels de PSPB dont la demi-vie est de 7 jours. C'est à cette condition que la détection des animaux non gravides (par absence de PSPB) pourra être pleinement satisfaisante. Le dosage de la PSPB sérique s'est avéré un excellent indicateur de mortalité embryonnaire (Humblot et al., 1988b), dont la fréquence est relativement élevée chez les vaches multipares (avant le $30^{\mathrm{e}}$ jour de gestation).

Malgré le grand intérêt pratique des PSP, nous ignorons, jusqu'à présent, leur rôle physiologique dans le déroulement d'une gestation.

EPF. - Bien que l'« Early Pregnancy Factor» (EPF) ne soit pas d'origine placentaire, mais issu de l'ovaire et de l'oviducte, nous ne pouvions l'omettre de ce texte en raison de sa précocité d'apparition par rapport à l'hCG ou les PSP au cours de la gestation. II fut découvert dans le sérum de souris gestante par Morton et al. (1976), puis chez la femme (Morton et al., 1977). Chez la brebis, $24 \mathrm{~h}$ après la fécondation, l'EPF est détectable dans le sérum (Morton et al., 1979). L'avortement, chez la femme et la brebis, entraîne la chute sérique de I'EPF en 24-48 h (Morton et al., 1977; Nancarrow et al., 1979). La reconnaissance de I'EPF est effectuée à l'aide d'un test s'appuyant sur sa propriété d'inhibition de rosettes (Noonan et al., 1979 ; Morton et al., 1982), qui sont les produits d'une hémagglutination entre les globules rouges et les lymphocytes d'espèces hétérologues. L'EPF réduit la quantité de sérum anti-lymphocytaire nécessaire à l'inhibition de rosettes. La conduite de ce test exige cependant un grand savoir-faire et présente une certaine complexité technique. Ainsi, la mise au point de tests plus performants, comme par exemple le test radioimmunologique, tant dans leur fiabilité que dans leur mise en œuvre, serait-elle un atout précieux pour la détection de ce facteur. Les diagnostics de gestation ou d'avortements précoces s'en trouveraient nettement améliorés.

EPAP. - Chez la génisse, une protéine sérique permet la discrimination entre animaux gravides ou non, dès les 8 premiers jours de gestation (Klima et al., 1987). Elle a été dénommée « Early Pregnancy Associated Protein » (EPAP), mais son origine trophoblastique et ses caractéristiques biochimiques et biologiques n'ont pas encore été déterminées.

$P L$ ou $C S$. - L'hormone lactogène placentaire humaine (hPL) présente, à I'heure actuelle, un intérêt mineur dans le diagnostic de grossesse, puisque sa détection est trop tardive (à partir de la $9^{\mathrm{e}}$ semaine). Sa sécrétion tout au long de la grossesse permet néanmoins de suivre le bon fonctionnement de l'unité fœto-placentaire et la détection d'éventuelles menaces d'avortement. La multiplicité de ses activités biologiques en font une molécule très intéressante pour l'étude des mécanismes de contrôle de la gestation. L'hPL fut partiellement 
purifiée en 1962 par Josimovich et Mac Laren à partir d'extraits bruts placentaires, puis fut isolée et bien caractérisée par diverses équipes (Cohen et al., 1964; Friesen, 1965 ; Florini et al., 1966). Sa séquence a été déterminée (Li et al., 1971 ; Sherwood et al., 1971). Cette holoprotéine comprend 191 acides aminés et présente une identité de $85 \%$ et une homologie de $96 \%$ avec l'hormone de croissance humaine (hGH) (Li et al., 1971). La biosynthèse de cette hormone a lieu dans le syncytiotrophoblaste (Sciarra et al., 1963; Friesen, 1973). L'hPL possède in vivo des propriétés somatotropes, mais nettement inférieures à celles de l'hormone de croissance hypophysaire (hGH). La mesure de l'activité somatotrope a été évaluée par l'augmentation du cartilage épiphysaire de tibia de rat hypophysectomisé (Greenspan et al., 1949). L'hPL, dans ce test, présente une activité de 12,8\% par rapport à l'hormone de croissance hypophysaire ( $\mathrm{Li}, 1970$ ). Son activité de croissance peut aussi être appréciée par l'incorporation de ${ }^{35} \mathrm{~S}$, in vivo et in vitro, par le cartilage costal de rat hypophysectomisé (Franchimont, 1965 ; Florini et al., 1966) ou par l'incorporation de thymidine tritiée dans le DNA du cartilage costal et dans la cellule adipeuse du rat hypophysectomisé (Murakawa et Raben, 1968; Breuer, 1969). Dans ces conditions expérimentales, l'activité de I'hPL est négligeable (1\% par rapport à l'hGH). La faible activité de croissance de l'hPL est confirmée par son infime liaison à des récepteurs hépatiques, d'origine humaine ou animale, révélateurs de l'activité somatotrope (Tsushima et Friesen, 1973).

En revanche, les propriétés lactogéniques et mammogéniques sont démontrées par de nombreux tests. Le premier a être utilisé fut celui du «jabot de pigeon ", dans lequel l'action de l'hPL provoque notamment l'augmentation de poids, ainsi qu'une hyperplasie de l'épithélium glandulaire (Josimovich et Mac Laren, 1962; Florini et al., 1966; Forsyth, 1967). Au cours d'une culture organotypique de glande mammaire de souris, I'hPL, associée à l'insuline et à l'hydrocortisone, stimule la synthèse de caséines, $d^{\prime} \alpha$-lactalbumine et de $\beta$ lactoglobuline (Turkington et Topper, 1966 ; Topper, 1970).

Cette intense activité lactogène et mammotrope se traduit également par une haute affinité de l'hPL aux récepteurs de glande mammaire, organe cible de l'hPL (Shiu et al., 1973).

Les propriétés lutéotrophiques de l'hPL ont été montrées chez le rat et la souris in vivo (Josimovich et al., 1963; Florini et al., 1966). In vitro, de la Llosa-Hermier et al. (1983) mirent en évidence un effet inhibiteur de l'hPL sur le catabolisme de la progestérone dans des ovaires de rattes pseudogestantes. Cette expérience confirme le rôle possible des hormones placentaires lactogènes au cours de la stéroïdogénèse (Tominaga et Troen, 1967) et particulièrement dans l'inhibition de la dégradation de la progestérone en $20 \alpha$-déhydro-progestérone (Josimovich et Archer, 1977). L'hPL semble intimement impliquée dans le métabolisme maternel afin d'assurer la croissance fœtale grâce à l'apport de nutriments. Récemment, Swenne et al. (1987) ont montré, à partir de cellules humaines de pancréas fœtal, que I'hPL était capable de stimuler la production d'insuline et d'un facteur de croissance analogue à l'insuline, I'IGF-I.

$L$ 'ensemble des hormones lactogènes placentaires ( $P L)$ ou somatomammotropines chorioniques (CS) de différentes espèces, ainsi que leurs propriétés de 
croissance et lactogènes est présenté dans le tableau 2. Les propriétés de l'oCS sont particulièrement analysées par ailleurs (Martal, 1980; Chêne et al., 1988).

$P A P P-A, P A P P-B$. - Les « pregnancy-associated plasma proteins » $\mathrm{A}$ et $\mathrm{B}$ (PAPP-A et PAPP-B) ont été détectées chez la femme (Lin et al., 1974, 1978a). La PAPP-A a été localisée dans le trophoblaste par Lin et Halbert, 1976), mais est produite également par l'endomètre (Duberg et al., 1982) et lors de tumeurs trophoblastiques (Walhström et al., 1981). Elle est présente à partir de la $5^{\mathrm{e}}$ semaine de grossesse. Il semble que ses fonctions biologiques possibles soit de type immunosuppressif (Kiefer, 1979; Bischof et al., 1982) et de type antiprotéasique (Bischof, 1980). Une des principales applications cliniques de la PAPP-A sert à l'évaluation du bon fonctionnement placentaire et du bon développement fœtal, selon Smith et al. (1980) et Hughes et al. (1980).

Malgré la détermination sérique de la PAPP-B, effectuée par Lin et al. (1978b), il n'existe pas de fonction connue à cette protéine.

\section{B. Protéines trophoblastiques impliquées dans le maintien de l'envi- ronnement progestéronique nécessaire au développement de l'em- bryon.}

Un environnement progestéronique adéquat est nécessaire à l'établissement et au maintien de toute gestation; plusieurs protéines placentaires ou extraplacentaires y contribuent.

Trophoblastine. - Parmi ces facteurs placentaires, nous avons déjà cité les gonadotrophines chorioniques et, à un moindre degré, I'hormone lactogène placentaire (de la Llosa-Hermier et al., 1983). Une protéine antilutéolytique a été mise en évidence : la trophoblastine. En 1966, Moor et Rowson avaient démontré que le conceptus (l'embryon et ses enveloppes) inhibait l'action lutéolytique cyclique d'origine utérine par un mécanisme local. Des injections utérines quotidiennes d'homogénats de trophoblastes ovins âgés de 14-16 jours, à des brebis cycliques dans la phase de sécrétion progestéronique, maintiennent les corps jaunes pendant plusieurs mois (Rowson et Moor, 1967 ; Martal et Lacroix, 1978 ; Martal et al., 1979). Par contre, l'injection de trophoblastes plus âgés (21 à 23 jours) ne permet pas le maintien des structures lutéales. Ce signal embryonnaire, de nature protéique, émis pendant une courte durée (12 à 22 jours chez la brebis), a été appelé trophoblastine. (Martal et al., 1979) et oTP1 (ovine trophoblast protein 1) (Godkin et al., 1984a).

Les caractéristiques physico-chimiques sont bien établies. Chez la brebis, c'est une protéine d'un poids moléculaire apparent de $20 \mathrm{kdal}$, déterminé par électrophorèse en gel d'acrylamide en système dénaturant, d'un point isoélectrique de 5,3, estimé par électrophorèse bidimensionnelle. La séquence de l'oTP1 a été déduite après clonage du DNA complémentaire (cDNA) (Imakawa et al., 1987). L'oTP1 présente une homologie de séquence de 45 à $55 \%$ avec les interférons de type humain, bovin, porcin, de rat et de souris. Des résultats analogues ont été simultanément obtenus dans notre laboratoire. La séquence 
amino-terminal de la trophoblastine a été élucidée; elle révèle une identité de $55 \%$ avec les interférons de classe I et de $64 \%$ avec l'interféron bovin $\alpha$ de classe II (Charpigny et al., 1988). Le c-DNA de la principale isoforme, l'isoforme 2 de la trophoblastine, a été cloné (Charlier et al., 1988).

Chez la vache, la présence de trophoblastine a été confirmée par des injections d'homogénats d'embryons bovins (Northey et French, 1980) et de vésicules trophoblastiques bovines (Heyman et al., 1984). Ces dernières, injectées à des brebis cycliques, permettaient également le maintien du corps jaune (Martal et al., 1984). Des vésicules trophoblastiques ovines injectées à des génisses cycliques, conduisaient au même résultat, ce qui suggère une parenté étroite entre trophoblastine ovine et bovine. II existe d'ailleurs une réaction immunologique croisée entre la trophoblastine ovine et bovine (Helmer et al., 1987). Récemment, pour mimer l'action de la trophoblastine, des injections intrautérines d'interféron $\alpha$ de classe I à des brebis (Flint et al., 1988) et à des vaches (Plante et al., 1988) cycliques ont permis d'observer le retard de la lutéolyse. La sécrétion d'oTP1 a été localisée histologiquement à l'aide d'anticorps spécifiques dans les cellules du trophectoderme (Godkin et al., 1984a). L'oTP1 présente une liaison spécifique aux membranes endométriales ovines. Bien que la sécrétion de la trophoblastine soit essentielle au maintien de la gestation chez les animaux, grâce à son effet antilutéolytique, elle n'a pu être détectée dans le sang des animaux gravides. La mise en évidence de son activité antivirale à l'aide d'un test très sensible (de l'ordre de $15 \mathrm{pg} / \mathrm{ml}$ de plasma) et la mise au point d'un dosage radioimmunologique sensible ont été réalisées (non publiés). Ces travaux confirment le rôle direct essentiellement local de la trophoblastine. Enfin, le fait que ce message embryonnaire soit un interféron embryonnaire l'implique également dans les mécanismes de la tolérance immunologique de la mère au moment de l'implantation du conceptus.

\section{Autres protéines de gestation.}

Le trophoblaste sécrète encore de très nombreuses molécules. Dans l'espèce humaine, un nombre considérable de protéines sériques d'origine placentaire a été caractérisé à des degrés divers. Nous avons présenté certaines d'entre elles : les protéines placentaires ou PP. Parmi elles, certaines sont d'origine extraplacentaire, comme la PP12 et la PP14, ainsi que diverses autres protéines de gestation que nous mentionnons ci-dessous.

Ainsi, la PP5, sécrétée par le syncytiotrophoblaste (Seppälä et al., 1979), a été mise en évidence par Bohn (1972) chez la femme et le singe (Bohn et Sedlacek, 1975). De par ses propriétés d'inhibition de protéases, cette protéine pourrait intervenir dans la régulation de l'invasion trophoblastique (Seppälä et al., 1979).

PP12-PP14. - Les glycoprotéines PP12 et PP14 ont été décrites par Bohn (Bohn et Kraus, 1980). La PP12 est sécrétée essentiellement par la décidua (Inaba et al., 1981). Cette protéine peut lier spécifiquement I'IGF-I (insulin-like growth factor I) et pourrait donc favoriser le transport sérique de ce facteur de 


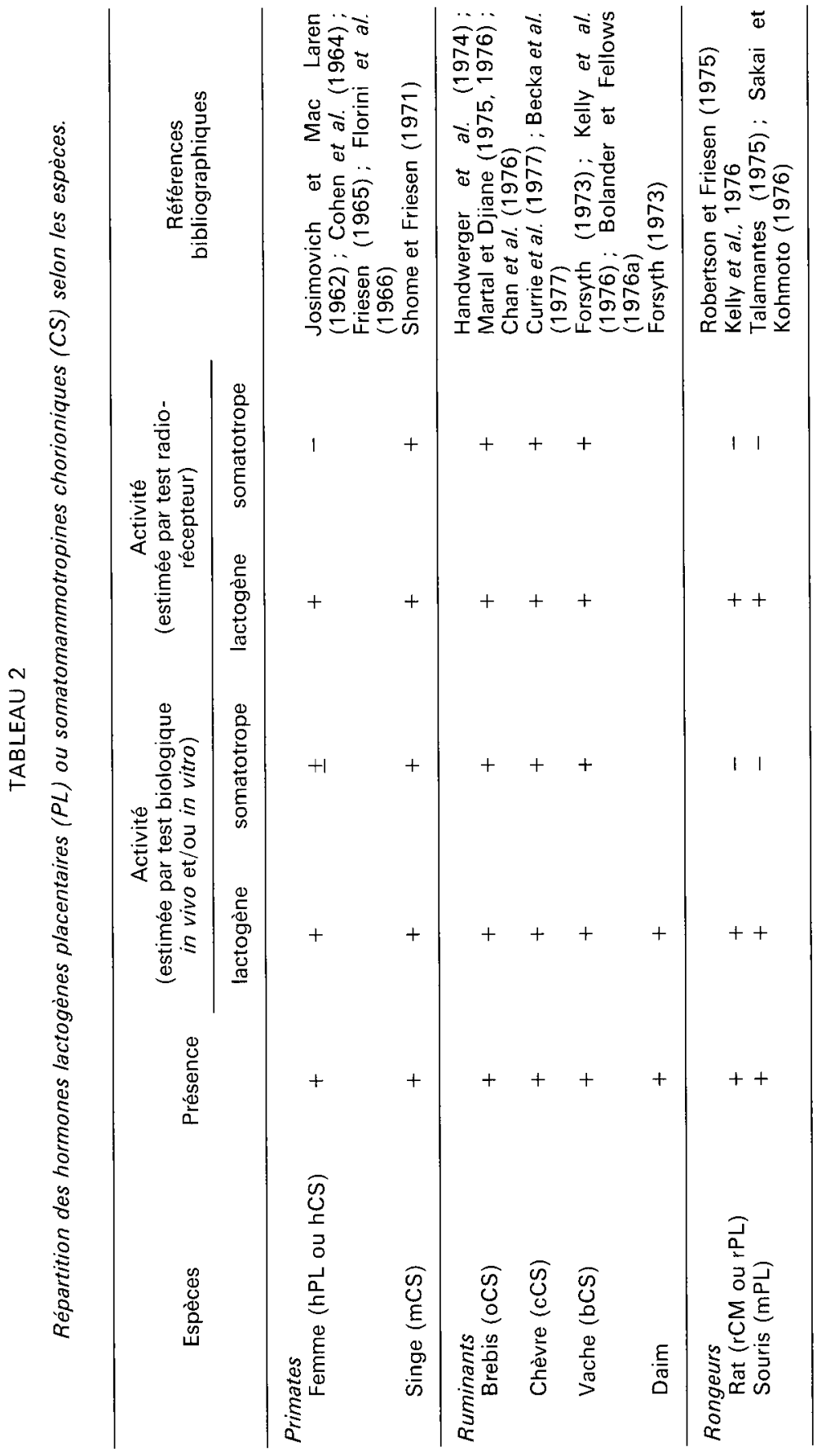




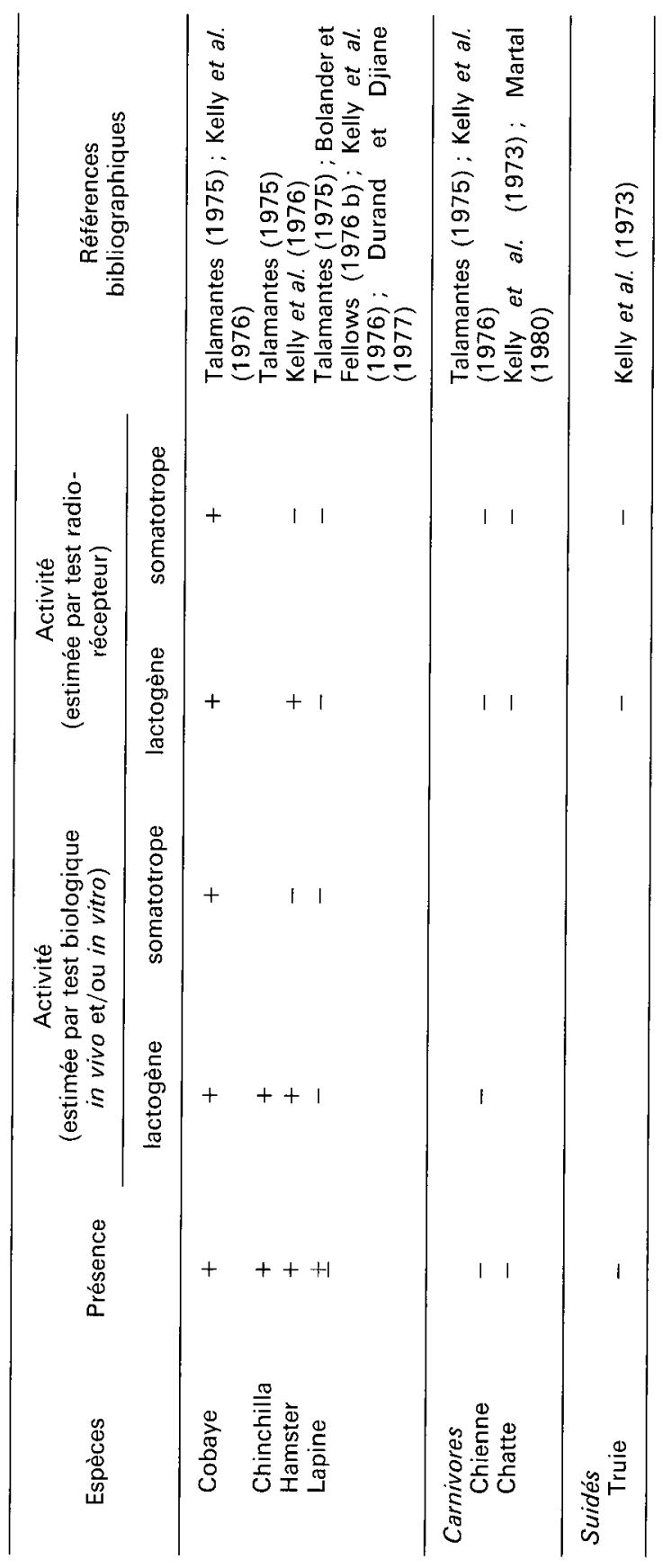


croissance et participer ainsi à la croissance fœtale (Petrunin et al., 1980, Koistinen et al., 1986). La glycoprotéine PP14 est, également, d'origine déciduale. On ne lui a, jusqu'à ce jour, pas encore attribué de fonction particulière.

Utéroglobuline. - Une protéine de gestation induite par la progestérone, uniquement chez la lapine, est retrouvée dans ses sécrétions utérines et synthétisée par l'endomètre (Beier, 1968). Elle a été dénommée utéroglobine ou blastokinine (Khrishnan et Daniel 1967). Elle représente la principale protéine de liaison de la progestérone (Beato, 1976).

$h p G H$. - Une hormone de croissance humaine a été extraite du placenta (Hennen et al., 1985). Elle se comporte comme un parfait agoniste de l'hormone de croissance hypophysaire en test de liaison à des récepteurs somatotropiques. Elle pourrait assurer, dans la seconde moitié de la grossesse, un rôle comparable à celui de l'hGH hypophysaire $22 \mathrm{~K}$ (Chêne et al., 1988).

Prolactine déciduale. - La décidua est également la source d'une prolactine que l'on retrouve dans le liquide amniotique chez les primates (Riddick et Kusmik, 1977). Son rôle physiologique reste à préciser, mais il a été impliqué dans l'osmorégulation de l'unité fœtoplacentaire.

Proliférine. - Une proliférine (PLF), molécule dont la structure est très proche de celle de la prolactine, est synthétisée par le placenta de souris, ainsi que par des cultures de cellules BALB/C 3 T3 en phase proliférative (Linzer et Nathans, 1984). Selon ces auteurs, la proliférine pourrait être assimilée à un facteur de croissance pour les tissus maternels et fotaux au cours de la gestation.

$\alpha$-fœetoprotéine $(A F P)$. - L' $\alpha$-fœetoprotéine est synthétisée essentiellement dans le foie fœtal et le sac vitellin et est présente dans de nombreuses espèces (Gitlin et Boesman, 1967). Elle est retrouvée en abondance dans les liquides fœtaux. Sa présence chez l'adulte est associée à une pathologie tumorale (Ruoslahti et Seppälä, 1979), d'où l'intérêt de sa détection. Son rôle dans le transport des acides gras lors de la maturation du tissu nerveux a été fortement suggéré.

Les protéines trophoblastiques ou d'origine utérine décrites dans ce texte participent au déroulement de la gestation dans ses différentes étapes, qui s'échelonnent de la transformation du corps jaune cyclique en corps jaune gestatif, à la différenciation et au développement embryonnaire et fœtal.

Cependant, il reste encore soit à approfondir la caractérisation biochimique de protéines pourtant identifiées, soit même à leur assigner une fonction biologique.

$27^{e}$ Réunion de la Société française pour l'Etude de la Fertilité Paris, 29, 30 sept., $1^{\text {er }}$ oct. 1988 .

\section{Références}

AHMED A. G., KLOPPER A., 1983. Diagnosis of early pregnancy by assay of placental proteins. $\mathrm{Br}$ J. Obstet. Gynaecol., 90, 604-611. 
AILENBERG M., SHEMESH M., 1983. Partial purification of a chorionic gonadotropin-like protein from bovine cotyledons. Biol. Reprod., 28, 517-522.

ASCHHEIM S., ZONDEK B., 1927. Hypophysenvorderlappen-hormon und Ovarialhormon im Harn von Schwangeren. Klin. Wschr., 6, 1322.

BAHL O. P., CARLSEN R. B., BELLISARIO R., SWAMINATHAN N., 1972. Human chorionic gonadotropin : amino acid sequence of the alpha and beta subunits. Bioch. Biophys. Res. Commun., 48, 416-422.

BARTOL F. F., ROBERTS R. M., BAZER F. W., LEWIS G. S., THATCHER W. W., 1985. Characterization of proteins produced in vitro by periattachment bovine conceptuses. Biol. Reprod., 32, $681-693$.

BEATO M., 1976. Binding of steroids to uteroglobin. J. Steroid Biochem., 7, 327-334.

BECKA S., BILEK J., SLABA J., SKARDA J., MIKULAS I., 1977. Some properties of the goat placental lactogen. Experimentia, 33, 771-772.

BEIER H. M., 1968. Uteroglobin : A hormone-sensitive endometrial protein involved in blastocyst development. Biochim. Biophys. Acta, 160, 189-291.

BISCHOF P., 1980. Observation on the isolation of macromolecules of placental origin, 47-55. In KLOPPER A., GENAZZANI A., CROSIGNANI P. G., The human placenta : proteins and hormones, Acad. Press, London and New York.

BISCHOF P., DUBERG S., SCHINDLER A. M., 1982. Is pregnancy-associated plasma protein A (PAPP-A) an immunomodulator during human pregnancy ? In KLOPPER A., Immunology of human placental proteins, Placenta, suppl. 4, 93-102 (Praeger New York)

BOHN H., 1971. Nachweis und Charakterisierung von Schwangerschaftsproteinen in der menschlichen Plazenta sowie ihre quantitative immunologische Bestimmung im Serum schwangerer Frauen. Arch. Gynaek., 210, 440-457.

BOHN H., 1972. Nachweis und Charakterisierung von höslichen Antigenen in der menschlichen Plazenta. Arch. Gynaek., 212, 165-175.

BOHN H., 1973. Detection, characterization and diagnostic significance of human pregnancyassociated glycoproteins. B/ut, 26, 205-209.

BOHN H., 1974. Immunochemical determinations of human pregnancy proteins. Arch. Gynaek., 217, 219-231.

BOHN H., 1985. Biochemistry of placental proteins. In BISCHOF P., KLOPPER A., Proteins of the placenta, 1-25, 5th Intern. Congress on placental proteins, Annecy (Karger, Basel, 1985).

BOHN H., KRAUS W., 1980. Isolierung und Charakterisierung eines neuen plazentaspezifischen Proteins (PP12). Arch. Gynecol., 229, 293-301.

BOHN H., KRAUS W., WINCKLER W., 1982. New soluble tissue proteins. In KLOPPER A., Immunology of human placental proteins, Placenta, Suppl. 4, 67-81 (Praeger, New York).

BOHN H., SEDLACEK H., 1975. Eine vergleichende Untersuchung von Plazentaspezifischen Proteinen bei Mensch und subhumanen Primaten. Arch. Gynaek., 220, 105-121.

BOLANDER F. F., FELLOWS R. E., 1976a. Purification and characterization of bovine placental lactogen. J. biol. Chem., 251, 2703-2708.

BOLANDER F. F., FELLOWS R. E., 1976b. The purification and characterization of rabbit placental lactogen. Biochem. J., 159, 775-782.

BREUER C. B., 1969. Stimulation of DNA synthesis in cartilage of hypophysectomized rats by native and modified placental lactogen and anabolic hormones. Endocrinology, 85, 989-999.

BUTLER J. E., HAMILTON W. C., SASSER R. G., RUDER C. A., HASS G. M., WILLIAMS R. J., 1982. Detection and partial characterization of two bovine pregnancy-specific proteins. Biol. Reprod., 26, 925-933.

CAMOUS S., CHARPIGNY G., GUILLOMOT M., MARTAL J., 1988. Purification of one bovine pregnancy-specific protein by high-performance liquid chromatography (HPLC). In Proc. int. Workshop on Maternal recognition of pregnancy and maintenance of the corpus luteum, Jerusalem (Israel) (abstr.)

CAVANAGH A. C., 1984. Production in vitro of mouse early pregnancy factor and purification to homogeneity. J. Reprod. Fert., 71, 581-592.

CHAN J. S. D., ROBERTSON H. A., FRIESEN H. G., 1976. The purification and characterization of ovine placental lactogen. Endocrinology, 98, 65-76. 
CHARLIER M., HUE D., MARTAL J., GAYE P., 1988. Molecular cloning cDNA structure and expression of ovine trophoblastin : identification as a class II $\alpha$ interferon Gene (sous presse).

CHARPIGNY G., REINAUD P., HUET J. C., GUILLOMOT M., CHARLIER M., PERNOLLET J. C., MARTAL J., 1988. High homology between a trophoblastic protein (trophoblastin) isolated from ovine embryo and $\alpha$-interferons. FEBS Letters, 228, 12-16.

CHÊNE N., MARTAL J., CHARRIER J., 1988. Ovine chorionic somatomammotropin and fœtal growth. Reprod. Nutr. Dévelop. 28, 1707-1730.

COHEN H., GRUMBACH M. M., KAPLAN S. L., 1964. Preparation of human chorionic "growthhormone-prolactin ». Proc. Soc. exp. Biol. Med., 117, 438-441.

COLE H. H., HART G. H., 1930. The potency of blood serum of mares in progressive stages of pregnancy in affecting the sexual maturity of the immature rat. Am. J. Physiol., 93, 57-68.

COMBARNOUS Y., SALESSE R., GARNIER J., 1981. Physico-chemical properties of pregnant mare serum gonadotropin. Bioch. Biophys. Acta, 667, 267-276.

CURRIE W. B., KELLY P.A., FRIESEN H.G., THORNBURN G. D., 1977. Caprine placental lactogen : levels of prolactin-like and growth hormone-like activities in the circulation of pregnant goats determined by radioreceptor assays. J. Endocr., 73, 215-226.

DE LA LLOSA-HERMIER M. P., LEBOULLEUX P., CHÊNE N., MARTAL J., 1983. Inhibitory effect of ovine and human placental lactogens on progesterone catabolism in luteinized rat ovaries in vitro. Placenta, 4, 479-488.

DUBERG S., BISHOF P., SCHINDLER A. M., BERUIN F., HERRMANN W., SIZONENKO P. C., 1982. Tissue and plasma concentrations of pregnancy associated plasma protein $A$ (PAPP-A) : comparison with other fetoplacental products. Br. J. Obstet. Gynaecol., 89, 352-357.

DURAND P., DJIANE J., 1977. Lactogenic activity in the serum of rabbits during pregnancy and early lactation. J. Endocr., 75, 33-42.

ETZRODT H., MUNSCH K., SCHRODER K. E., PFEIFFER E. F., 1980. Somatostatin-like activity in placenta, amniotic fluid and umbilical cord plasma. In KLOPPER A., GENAZZANI A., CROSIGNANI P. G., The human placenta, 272-283, Serono Symposium No. 35, Acad. Press, London.

FLINT A.P. F., STEWART H. J., LAMMING G. E., 1988. Interferons and the control of corpus luteum function in early pregnancy in ruminants. Int. Congr. Endocrinol., Tokyo (Japon).

FLORINI J. R., TONELLI F., BREUER C. B., COPPOLA J., RINGLER I., BELL P. H., 1966. Characterization and biological effects of purified placental protein (human). Endocrinology, 79. 692-708.

FORSYTH I.A., 1967. Prolactin and placental lactogen. In GRAY C. H., BACHARACH A. L., Hormones in blood, 233-272, Vol. I, Acad. Press, London-N.Y.

FORSYTH I.A., 1973. Secretion of a prolactin-like hormone by the placenta in ruminants. In DENAMUR R., NETTER A., Le corps jaune, 239-255, Masson, Paris.

FRANCHIMONT P., 1965. Présence d'une hormone de croissance dans le placenta. Ann. Endocr., 26, 346-355.

FRIESEN H. G., 1965. Purification of placental factor with immunological and chemical similarity to human growth hormone. Endocrinology, 76, 369-381.

FRIESEN H. G., 1973. Placental protein and polypeptide hormones. In GREEP R. O., ASTWOOD E. G., Female reproductive system, Part 2, 295-309, Handbook of physiology, section 7 : Endocrinology, Vol. II, Am. physiol. Soc., Washington, D.C.

GASPARD U., 1979. Les hormones protéiques placentaires. pp 364, Masson, Paris.

GENAZZANI A. R., HURLIMANN J., FIORETTI P., FELBER J.P., 1974. In vitro synthesis of an $\mathrm{ACTH}$-like hormone and human chorionic somatomammotrophin by placenta and amniotic cells. Experientia, 30, 430-432.

GIBBONS J. M., MITNIK M., CHIEFFO V., 1975. In vitro biosynthesis of TSH and LH-releasing factors by the human placenta. Am. J. Obstet. Gynec., 121, 127.

GITLIN D., BOESMAN M., 1967. Fetus-specific serum proteins in several mammals and their relation to human $\alpha$-fetoprotein. Comp. Biochem. Physiol., 21, 327-336. 
GODKIN J. D., BAZER F. W., MOFFATT J., SESSIONS F., ROBERTS R. M., 1982. Purification and properties of a major, low component weight protein released by the trophoblast of sheep blastocysts at day 13-21. J. Reprod. Fert. 65, 141-150.

GODKIN J. D., BAZER F.W., ROBERTS R. M., 1984a. Ovine trophoblast protein 1, an early secreted blastocyst protein binds specifically to uterine endometrium and affects protein synthesis. Endocrinology, 114, 120-130.

GODKIN J. D., BAZER F.W., THATCHER W.W., ROBERTS M., 1984b. Proteins released by cultured day 15-16 conceptuses prolong luteal maintenance when introduced into uterine lumen of cyclic ewes. J. Reprod. Fert., 71, 57-64.

GREENSPAN F. S., LI C. H., SIMPSON M. E., EVANS H. M., 1949. Bioassay of hypophyseal growth hormone: the tibia test. Endocrinology, 45, 455-463.

HANDWERGER S., MAURER W., BARRETT J., HURLEY T., FELLOWS R. E., 1974. Evidence for homology between ovine and human placental lactogen. Endocr. Res. Commun., 1. 403-413.

HAOUR F., SAXENA B. B., 1974. Detection of gonadotropin in the rabbit blastocyst before implantation. Science, 185, 444-445.

HAOUR F., TELL G., SANCHEZ P., 1976. Mise en évidence et dosage d'une gonadotrophine chorionique chez le rat. C.R. Acad. Sci. sér. D, 282, 1183-1186.

HELMER S. D., HANSEN P. J., ANTHONY R. V., THATCHER W. W., BAZER F. W., ROBERTS R. M., 1987. Identification of bovine trophoblast protein-1, a secretory protein immunologically related to ovine trophoblast protein-1. J. Reprod. Fert., 79, 83-91.

HENNEN G. P., 1965. Detection and study of a human chorionic-thyroid-stimulation factor. Archs int. Physiol., 73, 689-695.

HENNEN G., FRANKENNE F., CLOSSET J., GOMEZ F., PIRENS G., EL KHAYAT N., 1985. A human placental $\mathrm{GH}$ : increasing levels during second half of pregnancy with pituitary $\mathrm{GH}$ suppression as revealed by monoclonal antibody radioimmunoassays. Int. J. Fertil., 30 , 27-33.

HEYMAN Y., CAMOUS S., FEVRE J., MEZIOU W., MARTAL J., 1984. Maintenance of corpus luteum after uterine transfer of trophoblastic vesicles in cyclic cows and ewes. J. Reprod. Fert., 70, 533-540.

HO P.C., CHAN S.Y.M., TANG G.W.K. 1988. Diagnosis of early pregnancy by enzyme immunoassay of Schwangerschafts-protein 1. Fertil. Steril., 49. 76-80.

HODGEN G. D., NIEMEN W. J., TULLNER W.W., 1975. Duration of chorionic gonadotropin production by the placenta of the rhesus monkey. Endocrinology, 96, 789-791.

HORNE C. H.W., TOWLER C. M., PUGH-HUMPHREY R. G.P., THOMSON A. Q., BOHN H., 1976. Pregnancy specific 1-glycoprotein, a product of the syncytiotrophoblast. Experientia, 32, 1197-1199.

HUGHES G., BISCHOF P., WILSON G., SMITH R., KLOPPER A., 1980. Tests of fetal wellbeing in the third trimester of pregnancy. Br. J. Obstet. Gynaecol., 87, 650-656.

HUMBLOT P., CAMOUS S., MARTAL J., CHARLERY J., JEANGUYOT N., THIBIER M., SASSER R. G., 1988a. Diagnosis of pregnancy by radioimmunoassay of a pregnancy-specific protein B in the plasma of dairy cows. Theriogenology, 30, 257-267.

HUMBLOT P., CAMOUS S., MARTAL J., CHARLERY J., JEANGUYOT N., THIBIER M., SASSER R. G., 1988b. Pregnancy-specific protein B progesterone concentration and embryonic mortality during early pregnancy in dairy cows. J. Reprod. Fert., 83, 215-223.

IMAKAWA K., ANTHONY R. V., KAZEMI M., MAROTTI K. R., POLITES H. G., ROBERTS R. M., 1987. Interferon-like sequence of ovine trophoblast protein secreted by embryonic trophectoderm. Nature, 330, 377-379.

INABA N., RENK T., WEINMANN E., BOHN H., 1981. Immunohistological location of proteins related to pregnancy specific 1-glycoprotein (SP1) and placenta-specific tissue proteins (PP10, PP12) by human and cynomolgus monkey leukocytes. Acta Haematol., 66, 35-38.

IZHAR M., SHEMESH M., 1988. Partial purification of a substance with luteotropic activity from bovine fetal cotyledon. In Proc. int. Workshop on Maternal recognition of pregnancy and maintenance of the corpus luteum, Jerusalem (Israel).

JOSIMOVICH J. B., ARCHER D. F., 1977. The role of lactogenic hormones in the pregnant woman and the fetus. Am. J. Obstet. Gynecol., 129, 777-780.

Reproduction, Nutrition, Développement, $n^{\circ} 6 \mathrm{~B} / 88-10$ 
JOSIMOVICH J. B., ATWOOD B. L., GOSS D. A., 1963. Luteotrophic, immunologic and electrophoretic properties of human placental lactogen. Endocrinology, 73, 410-420.

JOSIMOVICH J. B., Mac LAREN J. A., 1962. Presence in the human placenta and term serum of a highly lactogenic substance immunochemically related to pituitary growth hormone. Endocrinology, 71, 209-220.

KELLY P. A., SHIU R. P. C., ROBERTSON M. C., FRIESEN M. G., 1973. Placental lactogen levels in several species throughout pregnancy. Endocrinology, 92, suppl., abstr. No. 370

KELLY P.A., TSUSHIMA T., SHIU R.P.C., FRIESEN H. G., 1976. Lactogenic and growth hormone-like activities in pregnancy determined by radioreceptor assays. Endocrinology, 99, 765-774.

KIEFER D. J., 1979. An investigation into the immunosuppressive properties of pregnancy serum. Ph. D. Thesis, Univ. Miami, USA.

KLIMA I., TIEMANN W., PITRA C., KAUFFOLD P., 1987. Serological detection of early pregnancy in cattle and partial characterization of a serum glycoprotein associated with early pregnancy. J. Reprod. Immunol., 11, 31 -39.

KLOPPER A., AHMED A. G., 1985. Subclinical abortion in unfertile women. In BISCHOF P., KLOPPER A., Proteins of the placenta, 114-122, 5th int. Congr. on placental proteins, Annecy (1984), Karger, Basel.

KOISTINEN R., KALKINEN N., HUHTALA M. L., SEPPALA M., BOHN H., RUTANEN E. M., 1986. Placental protein 12 is a decidual protein that binds somatomedin and has an identical $\mathrm{N}$-terminal amino acid sequence with somatomedin-binding protein from human amniotic fluid. Endocrinology, 118, 1375-1378.

KRISHNAN R. S., DANIEL J.C. Jr., 1967. "Blastokinin »: Inducer and regulator of blastocyst development in the rabbit uterus. Science, 158, 490-492.

LACROIX M. C., MARTAL J., 1979. Mise en évidence et évolution d'une gonadotrophine chorionique chez la brebis. C.R. Acad. Sci. Paris, Sér. D, 288, 771-774.

LI C. H., 1970. On the characterization of human chorionic somatomammotropin. Annali Sclevo., 12, $651-654$.

LI C. H., DIXON J. S., CHUNG D., 1971. Primary structure of the human chorionic somatomammotropin (hCG) molecule. Science, 173, 56-58.

LIN T. M., HALBERT S. P., 1976. Placental localisation of human pregnancy proteins. Science, 193, 1249-1252.

LIN T. M., HALBERT S. P., KIEFER D., 1978a. Characterization and purification of pregnancyassociated plasma protein B (PAPP-B). Int. Arch. Allergy appl. Immun., 57, 249-303.

LIN T. M., HALBERT S. P., KIEFER D., SPELLACY W. N., GALL S., 1974. Characterization of four human pregnancy-associated plasma proteins. Am. J. Obstet. Gynaec., 118, 223-236.

LIN T. M., HALBERT S. P., SPELLACY W. M., 1978b. Pregnancy-associated plasma protein B (PAPP B) in normal and abnormal pregnancies at term. Br. J. Obstet. Gynaecol., 85, 652-656.

LINZER D. I. H., NATHANS D., 1984. Nucleotide sequence of a growth-related mRNA encoding a member of the prolactin-growth hormone family. Proc. nat. Acad. Sci. USA, 81, 4255-4259.

MARTAL J., 1980. L'hormone lactogène placentaire ovine. Purification et propriétés physicochimiques, immunologiques et biologiques. Etude de son rôle dans les mécanismes de contrôle de la gestation. Th. Doct. ès Sci. d'Etat en Biologie, $499 \mathrm{p}$.

MARTAL J., CAMOUS S., FĖVRE J., CHARLIER M., HEYMAN Y., 1984. Specificity of embryonic signals maintaining corpus luteum in early pregnancy in ruminants. Proc. 10th int. Congr. on Anim. Reprod. Al, Urbana-Champaign, III., USA, No. 510, $3 \mathrm{p}$.

MARTAL J., DJIANE J., 1975. Purification of a lactogenic hormone in sheep placenta. Bioch. Biophys. Res. Comm., 65, 770-778.

MARTAL J., DJIANE J., 1976. Isolement d'une hormone placentaire ovine permettant la lactation et la croissance. // Journée de la recherche ovine et caprine INRA-ITOVIC, Paris, 322-329, ITOVIC-SPEOC, Paris.

MARTAL J., LACROIX M. C., 1978. Importance d'une trophoblastine dans le contrôle endocrinien du corps jaune gestatif chez la brebis au moment de l'implantation. In du MESNIL du BUISSON F., PSYCHOYOS A., THOMAS K., Implantation de l'ceuf, 193-208, Masson, Paris. 
MARTAL J., LACROIX M. C., LOUDES C., SAUNIER M., WINTENBERGER-TORRÈS S., 1979. Trophoblastin, an antiluteolytic protein present in early pregnancy in sheep. J. Reprod. Fert., 56, 63-73.

MIDGLEY A. R. Jr., PIERCE G. B., 1962. Immunohistochemical localization of human chorionic gonadotropin. J. Exp. Med., 115, 289-294.

MOOR R. M., ROWSON L. E. A., 1966. The corpus luteum of the sheep : functional relationship between the embryo and the corpus luteum. J. Endocr., 34, 233-239.

MORTON H., HEGH V., CLUNIE C. J. A., 1976. Studies of the rosette inhibition test in pregnant mice : evidence of immunosuppression? Proc. R. Soc. Lond. B, 193, 413-419.

MORTON H., NANCARROW C. D., SCARAMUZZI R. J., EVISON B. M., CLUNIE G. J. A., 1979. Detection of early pregnancy in sheep by the rosette inhibition test. J. Reprod. Fert., 56, 75-80.

MORTON H., ROLFE B. E., CAVANAGH A. C., 1982. Early pregnancy factor - biology and clinical significance. In GRUDZINSKAS J.G., TEISNER B., SEPPÄLÄ M., Pregnancy proteins, 391 - 406, Acad. Press, Sydney.

MORTON H., ROLFE B. E., CLUNIE G. J. A., ANDERSON M. J., MORRISSON J., 1977. An early pregnancy factor detected in human serum by the rosette inhibition test. Lancet, 1, 394-397.

MURAKAWA S., RABEN M., 1968. Effect of growth hormone and placental lactogen on DNA synthesis in rat costal cartilage and adipose tissue. Endocrinology, 83, 645-650.

NANCARROW C. D., EVISON B. M., SCARAMUZZI R. J., TURNBALL K. E., 1979. Detection of induced death of embryos in sheep by the rosette inhibition test. J. Reprod. Fert., 57. 385-389.

NANCARROW C. D., WALLACE A. L. C., GREWAL A. S., 1981. The early pregnancy factor of sheep and cattle. J. Reprod. Fert., Suppl. 30, 191-199.

NOONAN E. P., HALLIDAY W. J., MORTON H., CLUNIE G. J. A., 1979. Early pregnancy factor is immunosuppressive. Nature, 278, 649-651.

NORTHEY D. L., FRENCH L. R., 1980. Effect of embryo removal and intra-uterine infusion of embryonic homogenates on the lifespan of the bovine corpus luteum. J. anim. Sci., 50, 298-302.

PETRUNIN D. D., GRYAZNOVA I. M., PETRUNINA Y. A., TATARINOV Y. S., 1978. Comparative immunological and physiochemical characteristics of human $\alpha 1$ - and $\alpha 2$-microglobulin. Buyll. aksp. Biol. Med., 5, 600-602.

PETRUNIN D. D., KOZLJAEVA G. A., MESNJANKINA N. V., SHEVCHENKO O. P., 1980. Detection of chorionic $\alpha 2$-microglobulin in the endometrium in the secretory phase of the menstrual cycle and in the male sperm. Akush Ginek., 3, 22-24.

PLANTE C., HANSEN P. J., THATCHER W. W., 1988. Prolongation of luteal lifespan in cows by intrauterine infusion of recombinant bovine alpha-interferon. Endocrinology, 122, 2342-2344.

REIMERS I. J., SASSER R. G., RUDER C. A., 1985. Production of pregnancy specific protein B (PSPB) by bovine binucleate trophoblastic cells. Biol. Reprod., 32, suppl. 11, 65 (abstract).

RIDDICK D. H., KUSMIK W. H., 1977. Decidua : a possible source of amniotic fluid prolactin. Am. J. Obstet. Gynecol., 127, 187-190.

ROBERTSON M. C., FRIESEN H. G., 1975. The purification and characterization of rat placental lactogen. Endocrinology, 97, 621-629.

ROWSON L. E. A., MOOR R. M., 1967. The influence of embryonic tissue homogenate infused into the uterus on life-span of the corpus luteum in the sheep. J. Reprod. Fert., 13, 511-516.

RUOSLAHTI E., SEPPÄLÄ M., 1979. Alpha-fetoprotein in cancer and fetal development. $A d v$. Cancer. Res., 29, 275-346.

SAKAI S., KOHMOTO K., 1976. Binding of mouse chorionomammotropin to prolactin receptors in the mouse mammary gland. Endocrinol. jap., 23, 499-503.

SASSER R. G., CROCK J., RUDER-MONTGOMERY C. A., 1988. Description of pregnancyspecific protein B in cattle. In Proc. int. Workshop Maternal recognition of pregnancy and maintenance of the corpus luteum, Jerusalem (Israel).

SASSER R. G., RUDER C. A., IVANI K.A., 1985. Pregnancy detection in farm animals by radioimmunoassay of a pregnancy-specific protein in serum. Int. Meet. on Pregnancy proteins in animals, Copenhagen (Denmark). 
SASSER R. G., RUDER C. A., IVANI K. A., BUTLER J. E., HAMILTON W. C., 1986. Detection of pregnancy by radioimmunoassay of a novel pregnancy-specific protein in serum of cows and a profile of serum concentrations during gestation. Biol. Reprod., 35, 936-942.

SCIARRA J. J., KAPLAN S. L., GRUMBACH M. M., 1963. Localization of anti-human growth hormone serum within the human placenta: evidence for a human chorionic "Growth hormone-Prolactin \#. Nature (Lond.), 199, 1005-1006.

SEPPÄLÄ M., RUTANEN E. M., HEIKINHEIMO M., JALANKO H., ENGVALL E., 1978 . Detection of trophoblastic tumour activity by pregnancy-specific $\beta 1$-glycoprotein. Int. J. Cancer., 21. 265-267.

SEPPÄLÄ M., WALHSTROM T., BOHN H., 1979. Circulating levels and tissue localisation of placental protein five (PP5) in pregnancy and trophoblastic desease: absence of PP5 expression in the malignant trophoblast. Int J. Cancer, 24, 6-10.

SHERWOOD L. M., HANDWERGER S., Mac LAURIN W. D., LANNER M., 1971. The amino-acid sequence of human placental lactogen. Nature New Biol., 233, 59-61.

SHIBASAKI T., ODAIGRI E., SHIZUME K., LING N., 1982. Corticotropin-releasing factor-like activity in human placental extracts. J. clin. Endocr. Metab., 55, 384-396.

SHIU R. P. C., KELLY P. A., FRIESEN H. G., 1973. Radioreceptor assay for prolactin and other lactogenic hormones. Science, 180, 968-970.

SHOME B., FRIESEN H. G., 1971. Purification and characterization of monkey placental lactogen. Endocrinology, 89, $631-641$

SMITH R., COOPER W., THOMSON M.A. R., 1980. Pregnancy-associated plasma protein A. A mesure of placental ageing, 109-113. In KLOPPER A., GENAZZANI A., GROSIGNANI P. G., The human placenta : proteins and hormones. Acad. Press London \& N. York.

SWENNE I., HILL D. J., STRAIN A. J., MILNER R. D. G., 1987. Effects of human placental lactogen and growth hormone on the production of insulin and somatomedin $\mathrm{C} /$ insulin-like growth factor I by human fetal pancreas in tissue culture. J. Endocr., 113, 297-303.

TALAMANTES F., 1975. Comparative study of the occurrence of placental prolactin among mammals. Gen. comp. Endocr., 27, 115-121.

TATARINOV Y.S., 1978. Trophoblast-specific beta 1 -glycoprotein as a marker for pregnancy and malignancies. Gynecol. Obstet. Invest., 9, 65-97.

TOMINAGA T., TROEN P., 1967. Stimulation of aromatization in human placenta by human placental lactogen. J. clin. Invest., 46, 1124A.

TOPPER Y. J., 1970. Multiple hormone interactions in the development of mammary gland in vitro. Rec. Progr. Horm. Res., 26, 287-308.

TSUSHIMA T., FRIESEN H. G., 1973. Radioreceptor assay for growth hormone. J. clin. Endocrinol. Metab., 37, 334-336.

TURKINGTON R.W., TOPPER Y.J., 1966. Stimulation of casein synthesis and histological development of mammary gland by human placental lactogen in vitro. Endocrinology, 79, 175-181.

WALHSTRÖM T., TEISNER B., LEE J. N., GRUDZINSKAS J.G., SEPPÄLÄ M., FOLKERSEN J., 1981. Placental-associated plasma protein-A (PAPP-A, SP4) in trophoblastic tumors. Acta Pathol. Microbiol. Scand. Sect. A, 89, 65-66.

WILEY L. D., 1974. Presence of a gonadotropin on the surface of preimplanted mouse embryos. Nature, 252, 715-716.

WINTENBERGER-TORRES S., 1978. Rôle actif de l'embryon avant l'implantation. In du MESNIL du BUISSON F., PSYCHOYOS A., THOMAS K., Implantation de l'ouf, 181-192, Masson, Paris.

YAMAMOTO S., KWOK S. C. M., GREENWOOD F. C., BRYANT-GREENWOOD G. D., 1981 . Relaxin purification from human placental basal plates. J. clin. Endocr. Metab., 52, 601-604.

YOSHIMI T., STROTT C. A., MARSHALL J. R., LIPSETT M. B., 1969. Corpus luteum function in early pregnancy. J. clin. Endocr. Metab., 29, 225-229. 